\title{
Association of hospital and physician case volumes with cardiac monitoring and cardiotoxicity during adjuvant trastuzumab treatment for breast cancer: a retrospective cohort study
}

\author{
Nicolas J. Chin-Yee MD, Andrew T. Yan MD, Alexander Kumachev MSc, Dennis Ko MD MSc, \\ Craig Earle MD MSc, George Tomlinson PhD, Maureen E. Trudeau MD, Murray Krahn MD MSc, \\ Monika Krzyzanowska MD MPH, Raveen Pal MD, Christine Brezden-Masley MD PhD, \\ Scott Gavura BScPharm MBA, Kelly Lien BSc, Kelvin K.W. Chan MD MSc
}

Abstract

Background: Adjuvant trastuzumab is the standard of care for patients with HER2 overexpressing breast cancer, but use of trastuzumab may lead to cardiotoxicity. Our goal was to evaluate the relationship between hospital and physician case volume and cardiac outcomes in this population.

Methods: In this retrospective cohort study, we identified all female patients in Ontario with a breast cancer diagnosis in 2003-2009 who underwent treatment with trastuzumab through a provincial drug-funding program and linked these patients to administrative databases to ascertain patient demographics, treating hospital and physician characteristics, admissions to hospital, cardiac risk factors, cardiac imaging and comorbidities. Insufficient cardiac monitoring was defined as per the Canadian Trastuzumab Working Group guideline. Cardiotoxicity was defined as receiving fewer than 16 of 18 doses of trastuzumab because of heart failure admission, heart failure diagnosis or discontinuation of the drug after cardiac imaging. We constructed hierarchical multivariable logistic regression models to evaluate the effect of annual hospital volume, cumulative physician volume and treatment period on cardiac monitoring and cardiotoxicity.

Results: Of 3777 women treated by 214 oncologists at 68 hospitals, 918 (24.3\%) had insufficient cardiac monitoring and cardiotoxicity developed in 640 (16.9\%). Cardiotoxicity occurred in 389 (42.4\%) and 251 (8.8\%) patients in the insufficient- and sufficient-monitoring groups, respectively. Higher annual hospital and cumulative physician volumes, and more recent calendar period, were all independent predictors for decreased cardiotoxicity. Adjustment for rates of cardiac monitoring annulled the relationships between case volume and cardiotoxicity.

Interpretation: Greater hospital and physician case volumes are associated with reduced rates of trastuzumab-related cardiotoxicity, most likely because of better cardiac monitoring at higher volume centres.

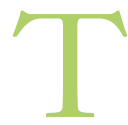
rastuzumab is a recombinant humanized monoclonal antibody against HER2, a transmembrane protein receptor that is overexpressed in $20 \%-25 \%$ of early stage breast cancers. ${ }^{1}$ It is clinically efficacious ${ }^{2-8}$ in the adjuvant setting at reducing disease recurrence and overall death in patients with HER2 overexpressing breast cancer and is currently considered the standard of care for this population. Trastuzumab, however, is associated with cardiotoxicity, especially when given in combination with taxanes or anthracyclines. ${ }^{5-7,9-11}$ A review of several clinical trials showed early discontinuation rates because of cardiotoxicity ranging from $4.6 \%$ to $19 \% .^{2}$ As such, baseline and regular cardiac monitoring is the standard of care during treatment with this agent. ${ }^{12}$

In oncology, studies reviewing the relationship between provider case volume and clinical outcomes have generally shown an inverse relationship between case volume and mor- tality in cancers for which treatment is primarily surgical. ${ }^{13-15}$ For breast cancer, higher hospital and surgeon volumes have been associated with increased postoperative survival. ${ }^{16-21}$ Understanding the relationship between case volume, cardiac monitoring and complication rates with for treatment with trastuzumab may have important practical implications for health care providers and policy-makers.

Competing interests: Andrew Yan received research funding from Roche. No other competing interests were declared.

This article has been peer reviewed.

Correspondence to: Kelvin Chan, kelvin.chan@sunnybrook.ca CMAJ Open 2016. DOI:10.9778/cmajo.20150033 
Accordingly, we used a population-based retrospective cohort of patients with breast cancer who were receiving adjuvant trastuzumab to evaluate the association between hospital and physician case volume, cardiac monitoring and cardiotoxicity.

\section{Methods}

\section{Study design and population}

This population-based retrospective cohort study included all female patients in Ontario with a diagnosis of breast cancer who were treated with adjuvant trastuzumab in 2003-2009. Those prescribed trastuzumab for metastatic breast cancer or other malignancies were excluded a priori from this cohort. We identified patients through the New Drug Funding Program, a publicly funded program that covers costs for newer drugs such as trastuzumab; therefore, we were able to capture nearly $100 \%$ of the patients receiving trastuzumab in Ontario during 2003-2009. Patient age, location of treatment, number of doses of trastuzumab and adjuvant chemotherapy regimen were obtained from the New Drug Funding Program database. This cohort was linked to other administrative databases located in the Institute for Clinical and Evaluative Sciences to ascertain socioeconomic status, information on imaging tests, patient comorbidities, and hospital admissions and discharges (Table S1 in Appendix 1, www.cmajopen.ca/content/4/1/E66/ suppl/DC1). The Research Ethics Board of Sunnybrook Health Sciences Centre approved this study.

\section{Explanatory variables and outcomes}

We studied hospital and physician case volumes as the main explanatory variables. Annual hospital and physician volumes were defined as the number of patients with breast cancer who were treated with adjuvant trastuzumab each year by a given hospital or physician. Cumulative physician volume was defined as the total number of patients, since 2003, who had been prescribed adjuvant trastuzumab by a physician at the onset of treatment. The case volume data were linked to the study participants by the hospitals where they were treated and the physicians who treated them for each year of diagnosis. For ease and clarity of presentation, we divided all case volume measures into terciles (i.e., each tercile contained about one-third of the patients), which were each characterized by the mean and SD of their yearly volumes.

We examined patient-level variables to identify potential confounders. These included age (grouped as $<45,45-64$ or $>64 \mathrm{yr}$ of age), medical comorbidities by Charlson-Deyo Inde ${ }^{22,23}(0-1$ or $\geq 2)$, previous diagnosis of heart failure, ${ }^{24}$ pre-existing hypertension, ${ }^{25}$ previous myocardial infarction, diabetes mellitus, ${ }^{26}$ income quintile, rural residence and calendar period of treatment. The 2 calendar periods compared were 2003-2007 and 2008-2009, because 2008 marked the introduction of updated practice guidelines for cardiac management during treatment with adjuvant trastuzumab. ${ }^{12}$

The 2 main outcome measures were adherence to cardiac monitoring guidelines (insufficient v. sufficient) and cardiotoxicity. A guideline from the Canadian Trastuzumab Working Group $^{12}$ recommends a minimum of 5 left ventricular ejection fraction assessments (echocardiogram or radionuclide ventriculography): 1 before initiation of trastuzumab treatment and then subsequent monitoring at months 3, 6, 9 and 12 during the course of treatment. In this study, insufficient cardiac monitoring was defined as lack of baseline cardiac imaging (from date of diagnosis to within $30 \mathrm{~d}$ after the first dose of trastuzumab), at least 1 fewer left ventricular ejection fraction assessment than recommended during trastuzumab treatment or a delay in cardiac monitoring by more than 1 month during treatment. ${ }^{12}$ We defined cardiotoxicity as receiving fewer than 16 of 18 doses of trastuzumab because the patient was admitted to hospital for heart failure, a new diagnosis of heart failure was captured by physician claims within 30 days of the last trastuzumab treatment or discontinuation of trastuzumab after cardiac imaging (left ventricular ejection fraction assessment within $30 \mathrm{~d}$ of last trastuzumab treatment).

\section{Statistical analyses}

We performed all statistical analyses using SAS (version 9.3). We conducted analyses using $\chi^{2}$ tests to compare the demographic and clinical features of patients who did and did not experience the 2 main outcomes. Patient characteristics were also compared between the hospital and physician volume terciles by $\chi^{2}$ tests.

Nested multivariable logistic regression models were used to assess the relationship of the outcome with annual hospital volume, cumulative physician volume and calendar period, with adjustment for potential risk factors. Model 1 included the variable of interest (annual hospital volume, cumulative physician volume or calendar period) and adjusted for the other 2 variables. Model 1 also adjusted for patient-level variables, which included age, comorbidities, previous heart failure, pre-existing hypertension, previous myocardial infarction, diabetes mellitus, income and rural residence. In addition, it accounted, by generalized linear mixed models, for physician clustering, hospital clustering and regional clustering based on regions defined by Local Health Integration Networks, the health authorities that fund and coordinate public health care services in Ontario. We fitted model 2 to assess whether the relationship between case volume and cardiotoxicity persisted when adjusting for levels of cardiac monitoring in addition to the model 1 variables.

We performed a series of sensitivity analyses (using model 1): the first analysis adjusted for 2-level (instead of 3-level) age grouping and used 55 years of age as a cut-off, the second analysis excluded patients with previous heart failure and the third analysis adjusted for anthracycline use (from time of diagnosis of breast cancer but before the start of adjuvant trastuzumab treatment) ascertained through physician claims.

We present the regression results as adjusted odds ratios (ORs) with 95\% confidence intervals (CIs) and $p$ values (values $<0.05$ were considered statistically significant).

\section{Results}

In total, 3777 patients with breast cancer in Ontario received adjuvant trastuzumab treatment from 2003 to 2009 and were treated at 68 centres by 214 physicians. Overall, 918 patients 


\section{OPEN}

Research

$(24.3 \%)$ did not receive sufficient cardiac monitoring and cardiotoxicity developed in 640 (16.9\%). Table 1 presents the demographic and clinical features of these patients and their association with cardiac monitoring and cardiotoxicity.

Multivariable models 1 and 2 fit with the observed data (Table S2 in Appendix 1). In model 1, the association between age and cardiotoxicity was no longer statistically significant, but increased comorbidity index (OR 1.49, 95\% CI 1.002.22) and previous heart failure diagnosis (OR 2.40, 95\% CI 1.23-4.67) both increased the risk of cardiotoxicity. After adjustment for the variables in model 1 , diabetes remained a significant predictor for insufficient monitoring (OR 1.47, 95\% CI 1.07-2.01), but nonrural residence became nonsignificant (OR 1.41, 95\% CI 0.98-2.02).

In univariable analysis, higher annual hospital volume and cumulative physician case volume, but not annual physician case volume, were associated with a decreased percentage of insufficient cardiac monitoring and decreased cardiotoxicity (Table S3, Appendix 1).
After accounting for the variables in model 1 , however, there was no significant relationship between hospital volume and cardiac monitoring (Table 2). Greater hospital volume remained an independent predictor for decreased cardiotoxicity in the full model ( $p=0.02$, Table 2$)$; cardiotoxicity was less likely to develop in patients who were treated in the combined second (OR 0.75, 95\% CI 0.56-1.01) and third (OR 0.72, 95\% CI 0.56-0.92) hospital-volume terciles than those treated in first-tercile hospitals.

In model 1, greater cumulative physician case volume was independently associated with sufficient cardiac monitoring $(p=0.01)$ and decreased cardiotoxicity $(p=0.05)$ (Table 3$)$. Patients who were treated in the second (OR $0.73,95 \%$ CI $0.57-0.93$ ) and third (OR 0.66, 95\% CI 0.49-0.90) physician case volume terciles were less likely to receive insufficient cardiac monitoring, and cardiotoxicity was also less likely to develop in these patients (tercile 2: OR 0.81, 95\% CI $0.65-$ 1.01; tercile 3: OR 0.73 , 95\% CI 0.56-0.96) compared with those in the first tercile. Annual physician case volume was

Table 1: Demographic and clinical features of patients with breast cancer who underwent treatment with adjuvant trastuzumab in Ontario from 2003 to $2009(n=3777)$

\begin{tabular}{|c|c|c|c|c|c|c|c|}
\hline \multirow[b]{2}{*}{ Characteristic } & \multirow[b]{2}{*}{$\begin{array}{c}\text { No. }(\%) \text { of } \\
\text { patients }\end{array}$} & \multicolumn{3}{|c|}{ Cardiac monitoring } & \multicolumn{3}{|c|}{ Cardiotoxicity } \\
\hline & & $\begin{array}{l}\text { No. }(\%) \text { of } \\
\text { patients with } \\
\text { sufficient } \\
\text { cardiac } \\
\text { monitoring } \\
n=2859\end{array}$ & $\begin{array}{c}\text { No. }(\%) \text { of } \\
\text { patients without } \\
\text { sufficient cardiac } \\
\text { monitoring } \\
n=918\end{array}$ & $p$ value $^{*}$ & $\begin{array}{c}\text { No. }(\%) \text { of } \\
\text { patients with } \\
\text { cardiotoxicity } \\
n=640\end{array}$ & $\begin{array}{c}\text { No. }(\%) \text { of } \\
\text { patients } \\
\text { without } \\
\text { cardiotoxicity } \\
n=3137\end{array}$ & $p$ value* \\
\hline Age, yr & & & & 0.3 & & & 0.02 \\
\hline$<45$ & 843 & $623(73.9)$ & $220(26.1)$ & & $123(14.6)$ & $720(85.4)$ & \\
\hline $45-64$ & 2312 & $1757(76.0)$ & $555(24.0)$ & & $392(17.0)$ & $1920(83.0)$ & \\
\hline$>64$ & 622 & $479(77.0)$ & $143(23.0)$ & & $125(20.1)$ & $497(79.9)$ & \\
\hline$\geq 2$ comorbidities & 172 & $123(71.5)$ & $49(28.5)$ & 0.2 & $42(24.4)$ & $130(75.6)$ & $<0.01$ \\
\hline Previous HF & 46 & $31(67.4)$ & $15(32.6)$ & 0.2 & $18(39.1)$ & $28(60.9)$ & $<0.01$ \\
\hline Hypertension & 1144 & $883(77.2)$ & $261(22.8)$ & 0.2 & $198(17.3)$ & $946(82.7)$ & 0.7 \\
\hline Previous MI & 13 & $9(69.2)$ & $4(30.8)$ & 0.6 & $4(30.8)$ & $9(69.2)$ & 0.2 \\
\hline Diabetes & 356 & $247(69.4)$ & $109(30.6)$ & $<0.01$ & $71(19.9)$ & $285(80.1)$ & 0.1 \\
\hline Income quintile & & & & 0.3 & & & 0.3 \\
\hline Q1 & 630 & $461(73.2)$ & $169(26.8)$ & & $120(19.0)$ & $510(80.9)$ & \\
\hline Q2 & 716 & $550(76.8)$ & $166(23.2)$ & & $127(17.7)$ & $589(82.3)$ & \\
\hline Q3 & 753 & $556(73.8)$ & $197(26.2)$ & & $117(15.5)$ & $636(84.5)$ & \\
\hline Q4 & 810 & $621(76.7)$ & $189(23.3)$ & & $142(17.5)$ & $668(82.5)$ & \\
\hline Q5 & 857 & $660(77.0)$ & $197(23.0)$ & & $134(15.6)$ & $723(84.4)$ & \\
\hline Rural & 472 & 396 (83.9) & $76(16.1)$ & $<0.01$ & $90(19.1)$ & $382(80.9)$ & 0.2 \\
\hline Calendar period & & & & 0.01 & & & $<0.01$ \\
\hline 2003-2007 & 2393 & $1780(74.4)$ & $613(25.5)$ & & 476 (19.9) & $1917(80.1)$ & \\
\hline 2008-2009 & 1384 & $1079(78.0)$ & $305(22.0)$ & & $164(11.8)$ & $1220(88.1)$ & \\
\hline
\end{tabular}


not a significant predictor for cardiac monitoring or cardiotoxicity in multivariable analysis (data not shown).

No important differences in the distributions of age, income or comorbidities existed between volume categories (Table S3, Appendix 1).

Of the $640(16.9 \%)$ patients who received a diagnosis of cardiotoxicity, 389 (60.8\%) also had insufficient cardiac monitoring. Although lower cumulative physician case volume was a predictor for both insufficient monitoring and cardiotoxicity in model 1, it was no longer associated with increased cardiotoxicity in model 2, which adjusted for cardiac monitoring (Table 3). Similarly, the significant relationship between annual hospital volume and cardiotoxicity disappeared after adjustment for cardiac monitoring (Table 2).

Patients treated in 2003-2007 had higher rates of insufficient monitoring and higher cardiotoxicity than those treated in 2008-2009 (Table 1), but after controlling for other variables, calendar period did not predict for insufficient cardiac monitoring (Table 4). Nonetheless, more recent treatment remained a significant independent predictor for decreased cardiotoxicity in the full model, even when adjusted for differences in cardiac monitoring (OR 0.54, 95\% CI 0.42-0.69; Table 4).

Multivariable sensitivity analyses that adjusted for a 2-level age grouping, excluded patients with a medical history of heart failure or adjusted for prior anthracycline use did not change the results (Tables S4-12, Appendix 1).

\section{Interpretation}

\section{Main findings}

In this large, population-based study involving patients with breast cancer receiving adjuvant trastuzumab treatment in Ontario, we showed that higher annual hospital volume and higher cumulative physician case volume were independent predictors for decreased trastuzumab-related cardiotoxicity rates, which were likely due to improved adherence to cardiac monitoring guidelines. We also observed decreased cardiotoxicity rates in recent years. In addition, we showed that trastuzumab-

Table 2: Annual hospital volume in relation to insufficient cardiac monitoring and cardiotoxicity in patients with breast cancer who underwent treatment with adjuvant trastuzumab in Ontario from 2003 to $2009(n=3777)$

\begin{tabular}{|c|c|c|c|c|}
\hline \multirow[b]{2}{*}{ Outcome } & \multicolumn{3}{|c|}{ Annual hospital volume, OR $(95 \% \mathrm{Cl})$} & \multirow[b]{2}{*}{$p$ value } \\
\hline & Tercile 1 & Tercile 2 & Tercile 3 & \\
\hline \multicolumn{5}{|c|}{ Insufficient monitoring } \\
\hline Model 1* & 1 (Reference) & $1.01(0.75-1.36)$ & $1.15(0.75-1.77)$ & 0.8 \\
\hline \multicolumn{5}{|c|}{ Cardiotoxicity } \\
\hline Model $1^{*}$ & 1 (Reference) & $0.72(0.56-0.92)$ & $0.75(0.56-1.01)$ & 0.02 \\
\hline Model 2† & 1 (Reference) & $0.93(0.68-1.27)$ & $0.74(0.49-1.12)$ & 0.3 \\
\hline \multicolumn{5}{|c|}{$\begin{array}{l}\text { Note: } \mathrm{OR}=\text { odds ratio, tercile } 1=\text { low, tercile } 2=\text { medium, tercile } 3=\text { high. } \\
\text { *Model } 1 \text { was adjusted for age, Charlson-Deyo index, previous heart failure, hypertension, previous myocardial infarction, } \\
\text { diabetes mellitus, income, rural residence, cumulative physician volume, calendar period of treatment, hospital clustering, } \\
\text { physician clustering and Local Health Integration Network clustering. } \\
\text { †Model } 2 \text { was adjusted for all variables in model } 1 \text { and further adjusted for sufficiency of cardiac monitoring. }\end{array}$} \\
\hline
\end{tabular}

Table 3: Cumulative physician case volume in relation to insufficient cardiac monitoring and cardiotoxicity in patients with breast cancer who underwent treatment with adjuvant trastuzumab in Ontario from 2003 to $2009(n=3777)$

\begin{tabular}{|c|c|c|c|c|}
\hline \multirow[b]{2}{*}{ Outcome } & \multicolumn{3}{|c|}{ Cumulative physician case volume, OR $(95 \% \mathrm{Cl})$} & \multirow[b]{2}{*}{$p$ value } \\
\hline & Tercile 1 & Tercile 2 & Tercile 3 & \\
\hline \multicolumn{5}{|c|}{ Insufficient monitoring } \\
\hline Model $1^{*}$ & 1 (Reference) & $0.73(0.57-0.93)$ & $0.66(0.49-0.90)$ & 0.01 \\
\hline \multicolumn{5}{|c|}{ Cardiotoxicity } \\
\hline Model $1^{*}$ & 1 (Reference) & $0.81(0.65-1.01)$ & $0.73(0.56-0.96)$ & 0.05 \\
\hline Model $2 \dagger$ & 1 (Reference) & $0.89(0.69-1.15)$ & $0.85(0.63-1.16)$ & 0.5 \\
\hline \multicolumn{5}{|c|}{$\begin{array}{l}\text { Note: } \mathrm{OR}=\text { odds ratio, tercile } 1=\text { low, tercile } 2=\text { medium, tercile } 3=\text { high. } \\
\text { *Model } 1 \text { was adjusted for age, Charlson-Deyo index, previous heart failure, hypertension, previous myocardial infarction, } \\
\text { diabetes mellitus, income, rural residence, annual hospital volume, calendar period of treatment, hospital clustering, physician } \\
\text { clustering and Local Health Integration Network clustering. } \\
\text { †Model } 2 \text { was adjusted for all variables in model } 1 \text { and further adjusted for sufficiency of cardiac monitoring. }\end{array}$} \\
\hline
\end{tabular}


related cardiotoxicity is common $(16.9 \%)$ in real-world patients with breast cancer, and elucidated some important predictive factors for insufficent cardiac monitoring and cardiotoxicity.

\section{Explanation and comparison with other studies}

The volume-outcome relationship has been reviewed for several different cancers. ${ }^{13-15}$ These studies, most of which used shortor long-term mortality as the outcome measure, yielded mixed results. The overall consensus held that an inverse relationship existed between case volume and mortality. In oncology, volume-outcome literature has largely focused on cancers for which treatment is primarily surgical, ${ }^{13-15}$ since outcomes of complex procedures may be more affected by case volume. ${ }^{13}$ Treatment at a higher volume, more specialized centre may also improve outcomes in adult ${ }^{13}$ and pediatric ${ }^{15}$ cancers for which treatment is primarily nonsurgical. For breast cancer, studies have consistently reported that higher hospital ${ }^{16-21}$ and surgeon $^{17,21}$ volumes are associated with increased postoperative survival, suggesting that centralization of surgical care for breast cancer to large specialized centres may be beneficial. We found a positive association between case volumes and the safe and effective delivery of systemic cancer treatment, which suggests that delivery of chemotherapy, particularly using novel agents with complex toxicity profiles, may also be enhanced through centralized care.

In our study, most of the patients who received a diagnosis of cardiotoxicity did not receive sufficient cardiac monitoring as recommended by the Canadian Trastuzumab Working Group guideline. ${ }^{12}$ Furthermore, adjustment for cardiac monitoring negated any significant associations between higher case volumes and cardiotoxicity. This suggests that trastuzumab-related cardiotoxicity may be a result of insufficient cardiac monitoring during treatment by less-experienced physicians. Of note, we found that cardiotoxicity was less likely to develop in those patients with more recent diagnoses and treatment (2008-2009)

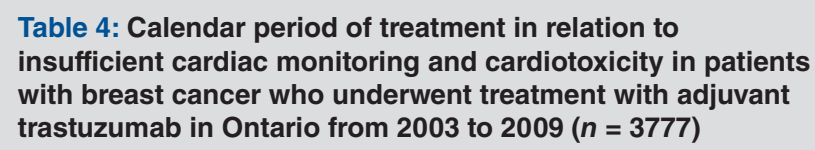

\begin{tabular}{|c|c|c|}
\hline \multirow[b]{2}{*}{ Outcome } & \multicolumn{2}{|c|}{ Calendar period, OR $(95 \% \mathrm{Cl})$} \\
\hline & 2003-2007 & 2008-2009 \\
\hline \multicolumn{3}{|l|}{$\begin{array}{l}\text { Insufficient } \\
\text { monitoring }\end{array}$} \\
\hline Model $1^{*}$ & 1 (Reference) & $0.93(0.74$ to 1.16$)$ \\
\hline \multicolumn{3}{|c|}{ Cardiotoxicity } \\
\hline Model $1^{*}$ & 1 (Reference) & $0.56(0.45$ to 0.70$)$ \\
\hline Model $2 \dagger$ & 1 (Reference) & $0.54(0.42$ to 0.69$)$ \\
\hline \multicolumn{3}{|c|}{$\begin{array}{l}\text { Note: OR = odds ratio. } \\
\text { *Model } 1 \text { was adjusted for age, Charlson Deyo index, previous heart failure, } \\
\text { hypertension, previous myocardial infarction, diabetes mellitus, income, rural } \\
\text { residence, annual hospital volume, cumulative physician volume, hospital } \\
\text { clustering, physician clustering and Local Health Integration Network clustering. } \\
\text { †Model } 2 \text { was adjusted for all variables in model } 1 \text { and further adjusted for } \\
\text { sufficiency of cardiac monitoring. }\end{array}$} \\
\hline
\end{tabular}

than in those treated earlier in the study period (2003-2007). This improvement in outcomes may be related to an increase in physicians' general awareness and experience with this treatment over time, perhaps in part because of the guideline. ${ }^{12}$ It may also be due to more careful patient selection or increased use of potentially cardioprotective medications, such as angiotensin-converting enzyme inhibitors and $\beta$-blockers. ${ }^{27-30}$

Our reported cardiotoxicity rate of $16.9 \%$ was found in an unselected cohort, thus substantiating the prevalence of this adverse effect in the general population with breast cancer. Indeed, recent retrospective studies ${ }^{9,11,31-33}$ reported higher rates of cardiotoxicity than those presented in clinical trials, $, 7,8,10,34,35$ likely because of the combined effects of more stringent patient selection and close monitoring for cardiac dysfunction in these trials. As expected, we found that a previous diagnosis of heart failure or a comorbidity index greater than 1 significantly predisposed patients to cardiotoxicity. In concordance with clinical trials, ${ }^{7,10,36-38}$ we observed increased toxicity with increasing age, although this trend became nonsignificant in the full multilevel model. In contrast with these trials, ${ }^{7,10,35}$ hypertension was not a significant risk factor for cardiotoxicity in our study. Two retrospective studies that focused on older patients receiving adjuvant trastuzumab treatment identified older age and cardiac comorbidities, including pre-existing hypertension and coronary artery disease, as risk factors for cardiotoxicity., ${ }^{9,31}$

\section{Limitations}

This study had several methodological strengths. Its population-based design allowed for a study involving an unselected group of patients with breast cancer. Mutual adjustment for patient and health care variables in a multilevel model was another strength. The major limitation of this study was that anthracycline use, specifically dosage, was not consistently captured in the administrative databases, thereby making it difficult to ascertain concurrent chemotherapy regimens.

In clinical trials and smaller retrospective cohort studies, cardiotoxicity is often defined by a decreased left ventricular ejection fraction from echocardiograms, a more sensitive but perhaps less clinicaly important definition. Given the large population-based nature of the present study, these data were not available. As with previous population-based studies, ${ }^{9}, 11,31$ our definition of cardiotoxicity includes a diagnosis of heart failure by physician claims but expands on this by including those patients who discontinued trastuzumab following cardiac imaging and those who did not complete a full course of treatment. The incorporation of trastuzumab discontinuation into our definition, although clinicially relevant, might have been affected by decisions to discontinue treatment unrelated to cardiac dysfunction. However, given the well-established efficacy and minimal noncardiac adverse effects of trastuzumab in clinical practice, it is rare to discontinue trastuzumab for any reason other than cardiotoxicity.

Because the universal coverage of oral medications by the Ontario Drug Benefit program is only available to older adults (65 yr of age or older), we were unable to adequately ascertain the use of cardiac medications in our population using the Ontario Drug Benefit database - an important consideration 
given that angiotensin-converting enzyme inhibitors and $\beta$-blockers have shown to be cardioprotective during chemotherapy. ${ }^{27-30}$ The prophylactic use of these medications during trastuzumab treatment, however, remains to be validated. ${ }^{39}$ Similarly, potential prognostic factors, such as left breast radiation ${ }^{40}$ and specific measures (e.g., left ventricular ejection fraction and blood pressure), were not available. Whereas many studies of volume effect evaluated overall survival, we deliberately focused on care processes rather than survival because of the nature of our study population, which comprised patients with early-stage breast cancer. Finally, since this is an observational study, we cannot establish causality. Nevertheless, large RCTs (patients randomly assigned to high v. low volume centres/physicians) are unlikely to be conducted in the future, and observational studies may provide valuable insights into care processes and outcomes in the real world.

\section{Conclusions and implications for practice and future research}

We showed that patients with breast cancer who were treated by experienced medical oncologists at high-volume cancer centres may have a lower risk of adverse effects during adjuvant trastuzumab treatment. Our study also confirmed that cardiotoxicity is a common adverse effect of trastuzumab treatment and supports the recommendation for regular cardiac assessments during treatment. The association between lower case volumes and cardiotoxicity could potentially be mitigated by centralization of care to high-volume centres or exploring barriers to cardiac monitoring at lower-volume sites. Given the high incidence of breast cancer and the appeal of providing treatment closer to home for patients, centralization of care may not be practical or desirable. Improving adherence to guidelines for cardiac monitoring will likely require a defined care pathway to monitor toxicity supported conjointly by nurses, pharmacists and oncologists. ${ }^{41}$ Furthermore, reimbursement linked to provision of cardiac test results at specific intervals during the course of therapy may improve compliance. Such actions to improve cardiac monitoring and knowledge translation in lower-volume centres may lead to better cardiac outcomes, irrespective of case volume.

\section{References}

1. Yamauchi H, Stearns V, Hayes DF. When is a tumor marker ready for prime time? A case study of c-erbB-2 as a predictive factor in breast cancer. 7 Clin Oncol 2001;19:2334-56

2. Mackey J, McLeod D, Ragaz J, et al. Adjuvant targeted therapy in early breast cancer. Cancer 2009;115:1154-68.

3. Dahabreh IJ, Linardou H, Siannis F, et al. Trastuzumab in the adjuvant treatment of early-stage breast cancer: a systematic review and meta-analysis of randomized controlled trials. Oncologist 2008;13:620-30.

4. Viani GA, Afonso SL, Stefano EJ, et al. Adjuvant trastuzumab in the treatment of her-2-positive early breast cancer: a meta-analysis of published randomized trials. BMC Cancer 2007;7:153.

5. Smith I, Procter M, Gelber RD, et al. 2-year follow-up of trastuzumab after adjuvant chemotherapy in HER2-positive breast cancer: a randomised controlled trial. Lancet 2007;369:29-36.

6. Slamon D, Eiermann $W$, Robert N, et al. Adjuvant trastuzumab in HER2-positive breast cancer. N Engl 7 Med 2011;365:1273-83.

7. Romond EH, Perez EA, Bryant J, et al. Trastuzumab plus adjuvant chemotherapy for operable HER2-positive breast cancer. N Engl 7 Med 2005;353:1673-84.

8. Piccart-Gebhart MJ, Procter M, Leyland-Jones B, et al. Trastuzumab after adjuvant chemotherapy in HER2-positive breast cancer. N Engl 7 Med 2005; 353:1659-72.
9. Chen J, Long JB, Hurria A, et al. Incidence of heart failure or cardiomyopathy after adjuvant trastuzumab therapy for breast cancer. 7 Am Coll Cardiol 2012; 60:2504-12

10. Romond EH, Jeong JH, Rastogi P, et al. Seven-year follow-up assessment of cardiac function in NSABP B-31, a randomized trial comparing doxorubicin and cyclophosphamide followed by paclitaxel (ACP) with ACP plus trastuzumab as adjuvant therapy for patients with node-positive, human epidermal growth factor receptor 2-positive breast cancer. 7 Clin Oncol 2012;30:3792-9.

11. Bowles EJ, Wellman R, Feigelson HS, et al. Risk of heart failure in breast cancer patients after anthracycline and trastuzumab treatment: a retrospective cohort study. 7 Natl Cancer Inst 2012;104:1293-305.

12. Mackey JR, Clemons M, Cote MA, et al. Cardiac management during adjuvant trastuzumab therapy: recommendations of the Canadian Trastuzumab Working Group. Curr Oncol 2008;15:24-35.

13. Hillner BE, Smith TJ, Desch CE. Hospital and physician volume or specialization and outcomes in cancer treatment: importance in quality of cancer care. $\mathcal{F}$ Clin Oncol 2000;18:2327-40.

14. Gruen RL, Pitt V, Green S, et al. The effect of provider case volume on cancer mortality: systematic review and meta-analysis. CA Cancer 7 Clin 2009;59:192-211.

15. Knops RR, van Dalen EC, Mulder RL, et al. The volume effect in paediatric oncology: a systematic review. Ann Oncol 2013;24:1749-53.

16. Roohan PJ, Bickell NA, Baptiste MS, et al. Hospital volume differences and five-year survival from breast cancer. Am 7 Public Health 1998;88:454-7.

17. Skinner KA, Helsper JT, Deapen D, et al. Breast cancer: Do specialists make a difference? Ann Surg Oncol 2003;10:606-15.

18. Guller U, Safford S, Pietrobon R, et al. High hospital volume is associated with better outcomes for breast cancer surgery: analysis of 233,247 patients. World 7 Surg 2005;29:994-9.

19. Peltoniemi P, Peltola M, Hakulinen T, et al. The effect of hospital volume on the outcome of breast cancer surgery. Ann Surg Oncol 2011;18:1684-90.

20. Vrijens F, Stordeur S, Beirens K, et al. Effect of hospital volume on processes of care and 5-year survival after breast cancer: a population-based study on 25000 women. Breast 2012;21:261-6.

21. Gooiker GA, van Gijn W, Post PN, et al. A systematic review and meta-analysis of the volume-outcome relationship in the surgical treatment of breast cancer. Are breast cancer patients better of with a high volume provider? Eur $\mathcal{F}$ Surg Oncol 2010;36(Suppl 1):S27-35.

22. Charlson ME, Pompei P, Ales KL, et al. A new method of classifying prognostic comorbidity in longitudinal studies: development and validation. $\mathcal{F}$ Chronic Dis 1987;40:373-83.

23. Deyo RA, Cherkin DC, Ciol MA. Adapting a clinical comorbidity index for use with ICD-9-CM administrative databases. 7 Clin Epidemiol 1992;45:613-9.

24. Schultz SE, Rothwell DM, Chen Z, et al. Identifying cases of congestive heart failure from administrative data: a validation study using primary care patient records. Chronic Dis Inj Can 2013;33:160-6.

25. Tu K, Chen Z, Lipscombe LL. Prevalence and incidence of hypertension from 1995 to 2005: a population-based study. CMA7 2008;178:1429-35.

26. Hux JE, Ivis F, Flintoft V, et al. Diabetes in Ontario: determination of prevalence and incidence using a validated administrative data algorithm. Diabetes Care 2002;25:512-6.

27. Kalay N, Basar E, Ozdogru I, et al. Protective effects of carvedilol against anthracycline-induced cardiomyopathy. 7 Am Coll Cardiol 2006;48:2258-62.

28. Silber JH, Cnaan A, Clark BJ, et al. Enalapril to prevent cardiac function decline in long-term survivors of pediatric cancer exposed to anthracyclines. 7 Clin Oncol 2004;22:820-8.

29. Cardinale D, Colombo A, Sandri MT, et al. Prevention of high-dose chemotherapy-induced cardiotoxicity in high-risk patients by angiotensin-converting enzyme inhibition. Circulation 2006;114:2474-81

30. Bosch X, Rovira M, Sitges M, et al. Enalapril and carvedilol for preventing chemotherapy-induced left ventricular systolic dysfunction in patients with malignant hemopathies: the OVERCOME trial (preventiOn of left Ventricular dysfunction with Enalapril and caRvedilol in patients submitted to intensive ChemOtherapy for the treatment of Malignant hEmopathies). 7 Am Coll Cardiol 2013;61:2355-62.

31. Chavez-MacGregor M, Zhang N, Buchholz TA, et al. Trastuzumab-related cardiotoxicity among older patients with breast cancer. 7 Clin Oncol 2013;31:4222-8.

32. Naumann D, Rusius V, Margiotta C, et al. Factors predicting trastuzumabrelated cardiotoxicity in a real-world population of women with HER2+ breast cancer. Anticancer Res 2013;33:1717-20.

33. Farolfi A, Melegari E, Aquilina M, et al. Trastuzumab-induced cardiotoxicity in early breast cancer patients: a retrospective study of possible risk and protective factors. Heart 2013;99:634-9.

34. Suter TM, Procter M, van Veldhuisen DJ, et al. Trastuzumab-associated cardiac adverse effects in the herceptin adjuvant trial. 7 Clin Oncol 2007;25:3859-65.

35. Procter M, Suter TM, de Azambuja E, et al. Longer-term assessment of trastuzumab-related cardiac adverse events in the Herceptin Adjuvant (HERA) trial. 7 Clin Oncol 2010;28:3422-8.

36. Tan-Chiu E, Yothers G, Romond E, et al. Assessment of cardiac dysfunction in a randomized trial comparing doxorubicin and cyclophosphamide followed by paclitaxel, with or without trastuzumab as adjuvant therapy in node-positive, human epidermal growth factor receptor 2-overexpressing breast cancer: NSABP B-31.7 Clin Oncol 2005;23:7811-9. 
37. Russell SD, Blackwell KL, Lawrence J, et al. Independent adjudication of symptomatic heart failure with the use of doxorubicin and cyclophosphamide followed by trastuzumab adjuvant therapy: a combined review of cardiac data from the National Surgical Adjuvant breast and Bowel Project B-31 and the North Central Cancer Treatment Group N9831 clinical trials. 7 Clin Oncol 2010;28:3416-21.

38. Perez EA, Suman VJ, Davidson NE, et al. Cardiac safety analysis of doxorubicin and cyclophosphamide followed by paclitaxel with or without trastuzumab in the North Central Cancer Treatment Group N9831 adjuvant breast cancer trial. 7 Clin Oncol 2008;26:1231-8.

39. Pituskin E, Haykowsky M, Mackey JR, et al. Rationale and design of the Multidisciplinary Approach to Novel Therapies in Cardiology Oncology Research Trial (MANTICORE 101-Breast): a randomized, placebo-controlled trial to determine if conventional heart failure pharmacotherapy can prevent trastuzumab-mediated left ventricular remodeling among patients with HER2+ early breast cancer using cardiac MRI. BMC Cancer 2011;11:318.

40. Fried G, Regev T, Moskovitz M. Trastuzumab-related cardiac events in the treatment of early breast cancer. Breast Cancer Res Treat 2013;142:1-7.

41. Kinsman L, Rotter T, James E, et al. What is a clinical pathway? Development of a definition to inform the debate. BMC Med 2010;8:31.

Affiliations: University of Toronto (Chin-Yee, Yan, Kumachev, Ko, Tomlinson, Trudeau, Krahn, Brezden-Masley, Chan); St. Michael's Hospital (Yan, Brezden-Masley); Institute for Clinical and Evaluative Sciences (Ko, Earle); Sunnybrook Health Sciences Centre (Ko, Earle, Trudeau, Chan); University Health Network (Tomlinson, Krzyzanowska), Toronto, Ont.; Kingston General Hospital (Pal), Kingston; Cancer Care Ontario
(Gavura); Canadian Centre for Applied Research in Cancer Control (Chan), Toronto, Ont.

Contributors: Kelvin Chan, Andrew Yan, Craig Earle, Maureen Trudeau and Scott Gavura conceived the study. Nicolas Chin-Yee drafted the manuscript. All of the authors contributed to data acquisition and/or analysis and interpretation of data, revised the article critically for important intellectual content, approved the final version to be published and agreed to act as guarantors of the work.

Funding: This study was funded by the Canadian Cancer Society (grant no. 700839) and was supported through provision of data by the Institute for Clinical and Evaluative Sciences and Cancer Care Ontario, and through funding support to the Institute for Clinical and Evaluative Sciences from an annual grant by the Ministry of Health and Long-Term Care and the Ontario Institute for Cancer Research. The opinions, results, and conclusions reported in this paper are those of the authors and are independent from the funding sources. No endorsement by the Institute for Clinical and Evaluative Sciences, Cancer Care Ontario, the Ontario Institute for Cancer Research or the Government of Ontario is intended or should be inferred.

Supplemental information: For reviewer comments and the original submission of this manuscript, please see www.cmajopen.ca/content/4/1/ E66/suppl/DC1 